\title{
Amplitude discrimination of sinusoids and narrow-band noise with Rayleigh properties
}

\author{
MICHAEL J. HAUTUS and R. JOHN IRWIN \\ The University of Auckland, Auckland, New Zealand
}

\begin{abstract}
The ability of human observers to discriminate differences in the amplitude of sinusoids and narrow-band noises was measured by the rating method of detection theory. Although each sinusoid (always $1000 \mathrm{~Hz}$ ) was presented at a fixed amplitude, its amplitude on any trial was drawn from one of two Rayleigh probability distributions that differed in mean amplitude: a signal distribution and a noise distribution. Similarly, the amplitudes of the narrow-band noises were distributed as the Rayleigh distribution by virtue of the reciprocal relation between their bandwidth $(100 \mathrm{~Hz}$ centered on $1000 \mathrm{~Hz})$ and duration $(10 \mathrm{msec})$. The obtained psychometric functions showing the area under the ROC as a function of signal-to-noise ratio were similar for both kinds of signals and were displaced, on average, about $4 \mathrm{~dB}$ from an ideal observer's function. The slopes of the obtained functions were similar to those of an ideal observer using 1 degree of freedomhalf the number available in Rayleigh noise.
\end{abstract}

When asked to discriminate small differences in the amplitude of two sounds, human observers do not perform at the same level as an ideal observer that makes the best possible use of the information available in the waveforms. There is nothing remarkable in this, but the exact nature of the information not used by human observers is an open question. One possibility is that the discrepancy between ideal and human observers depends upon the waveform's bandwidth. For wide-band noise, Green (1960) and Irwin (1989) reported a discrepancy of 5-7 dB between ideal and human observers, but for an approximation to narrow-band noise, Ronken (1969) reported a discrepancy of only $0.7 \mathrm{~dB}$.

Ronken's (1969) results are important because he studied a special case of narrow-band noise known as Rayleigh noise. A band-limited noise waveform can be represented by a finite number of equally spaced discrete samples and, according to Shannon's sampling theorem, the number of samples required is equal to $2 W T$, where $W$ is the bandwidth of the noise in hertz and $T$ is its duration in seconds. For Rayleigh noise, the duration is the reciprocal of the bandwidth and, therefore, $2 W T=2$. This is a limiting case because every additional cycle of the noise's bandwidth requires two additional samples to represent it (Green \& McGill, 1970). Furthermore, the energy in such a waveform is distributed as chi square with $2 W T=2 \mathrm{df}$. The amplitude of the waveform is dis-

This report is based in part on doctoral research being undertaken by the first author. The research was supported by grants from the Auckland University Research Committee. We thank the National Audiology Centre, Auckland, for the loan of some equipment, and Donald Laming for his comments on an earlier version of the manuscript. Requests for reprints should be sent to R. J. Irwin, Department of Psychology, The University of Auckland, Private Bag 92019, Auckland, New Zealand. tributed as the Rayleigh distribution, a distribution that is a transformation of the chi-square distribution with $2 \mathrm{df}$ (Egan, 1975). If $A$ is the amplitude of a brief sample, and $\sigma^{2}$ is the variance of the Gaussian noise source, then the density function of the amplitudes is given by:

$$
f(A)=\left(1 / \sigma^{2}\right) \exp \left(-A^{2} / \sigma^{2}\right) \quad(A>0) .
$$

Ronken's (1969) stimuli, however, differed from true Rayleigh noise in a crucial way: they were sinusoids of fixed amplitude, not noise waveforms. The amplitudes of the sinusoids were sampled from one of two Rayleigh distributions that differed in mean value. Ronken reasoned that Rayleigh noise of sufficiently narrow bandwidth could be characterized as a sinusoid with slowly varying amplitude and phase (see, e.g., Green \& Swets, 1966). Therefore, a brief sample of the waveform could be approximated by a sinusoid of constant amplitude. This approximation may be critical, because Whitmore, Williams, and Ermey (1968) studied the discrimination of noise bursts for which $2 W T=2$. In contrast to Ronken, they found a discrepancy of the order of 3-6 dB between the performance of their observers and the theoretical ideal for Rayleigh noise.

Because Ronken's (1969) result is so close to the ideal, and because it differs from Whitmore et al.'s (1968) result for true Rayleigh noise, these findings deserve further study. This investigation had three objectives. First, we compared psychometric functions for amplitude discrimination from the same observers for both Rayleighdistributed sinusoids and narrow-band Rayleigh noise. The questions we hoped to answer were (1) whether Ronken's remarkable finding that human observers performed at near-ideal levels could be replicated, and (2) whether the two versions of Rayleigh noise we used would yield the same results when studied by the same methods with the same observers. 
Second, we obtained receiver operating characteristics (ROCs) for each signal. Our purpose here was to compare the shape and location of the obtained ROCs with those predicted for an ideal observer on the task. We reasoned that the shape of the ROC, in addition to the index of detectability that can be derived from the ROC, might indicate the way in which human observers differ from their ideal counterpart. To obtain ROCs efficiently, we followed Whitmore et al.'s (1968) procedure and employed the rating method of signal detection rather than the forced-choice method that Ronken (1969) adopted.

Third, we checked to see if detectability of these signals was independent of their average level; in other words, whether Weber's law held for this task. The decline in the Weber fraction with amplitude is known as the near miss to Weber's law. Ronken (1969) had found that Weber's law, rather than the near miss to Weber's law, held for these waveforms despite the fact that the near miss to Weber's law is normally found for amplitude resolution of sinusoids. The usual finding is that the Weber fraction for sinusoids decreases with their amplitude, rather than remaining independent of amplitude as Weber's law prescribes. A valuable summary of the evidence for this phenomenon is provided by Laming (1986).

We thought it unlikely that we would observe the near miss for this task, even though our stimuli included sinusoids. The reason, as Ronken (1969) noted, is that the variability in amplitude of a Rayleigh process is large relative to the normal amplitude resolution of the human ear. For example, Jesteadt, Wier, and Green (1977) found that the Weber fraction for a $1000-\mathrm{Hz}$ sinusoid, expressed as a ratio of intensities, declined from 0.354 at $20 \mathrm{~dB}$ SL to 0.099 at $80 \mathrm{~dB} \mathrm{SL}$. When expressed as a ratio of amplitudes, these Weber fractions decline from 0.177 to 0.055 . In contrast, the ratio of the standard deviation of a Rayleigh distribution to its mean ${ }^{1}$-its coefficient of variation-can be shown to be $\sqrt{(4 / \pi-1)}$, which is 0.523 . This ratio of amplitudes can be taken as the Weber fraction for such a stimulus (Woodworth \& Schlosberg, 1954, p. 199), and it exceeds the Weber fraction for amplitude resolution of two sinusoids at the sound pressures we employed. Thus, we expected that the variability in amplitude of the signals themselves would overwhelm the amplitude resolution of the ear and, therefore, that the near miss to Weber's law would not be seen.

\section{EXPERIMENT 1}

\section{Method}

\section{Observers}

Three subjects (including the first author) served as observers. All were experienced at psychophysical tasks, and all undertook several thousand practice trials before undertaking the experimental observations.

\section{Apparatus}

Sinusoids. To replicate Ronken's (1969) experiment, sinusoids of $1000 \mathrm{~Hz}$ with Rayleigh-distributed amplitudes were generated by a waveform synthesizer (Hewlett-Packard, Model 8904A). Each sinusoid was gated with a $10-\mathrm{msec}$ Hanning function and was presented for $100 \mathrm{msec}$. The probability of selecting any amplitude was determined by dividing a Rayleigh probability density function into 20 equally spaced bins. The amplitude in the center of a bin was presented with a probability equal to the area of the bin under the density function. ${ }^{2}$

Narrow-band noise. Rayleigh noise was produced by gating and filtering a wide-band Gaussian source (General Radio analogue noise generator, Model 1381). The equivalent rectangular bandwidth of the filter, centered at $1000 \mathrm{~Hz}$, was $100 \mathrm{~Hz}$ (Wavetek "Brickwall" filter, Model 753A), and the equivalent rectangular duration of the waveform was $10 \mathrm{msec}$; thus, $2 W T=2$. The filter was placed after the gate; therefore, the waveform's duration was longer than the nominal duration for which the gate was open. To ensure that the noise had Rayleigh properties, the statistics of the energy content of the bursts were computed and compared with those of the chisquare distribution with $2 \mathrm{df}$. To do this, the voltage waveform of each burst was sampled at a rate of $20 \mathrm{kHz}$ in order to determine its mean square voltage. The probability distribution function of these mean square voltages, standardized to a mean of 2 , was compared with the distribution function of chi square with $2 \mathrm{df}$ by means of the Kolmogorov-Smirnov test. Figure 1 shows the chi-square distribution function and an example of an obtained function for 1,021 noise samples. In this example, the Kolmogorov-Smirnov statistic, $D$, is 0.033 ( $p>.05$ ).

To mask any transients, a second noise generator provided a 7 $\mathrm{kHz}$ band of masking noise that was present throughout both experiments. Its overall level was always $40 \mathrm{~dB}$ SPL, which is equivalent to a spectral level of $1.5 \mathrm{~dB}$. The stimuli were presented monaurally by earphones (TDH-49 in MX 41/AR cushions).

\section{Nomenclature}

In specifying signal-to-noise ratios, we adopted Egan's (1975) notation. The RMS voltage of Gaussian noise of zero mean voltage is equal to its standard deviation, $\sigma$. The average power of the noise across a $1-\Omega$ resistor is equal to its squared RMS voltage, $\sigma^{2}$. We used the subscript $n$ to designate the noise power, $s$ to designate the signal power, and $s n$ to designate the signal-plus-noise power. Thus, $\sigma_{s n}^{2}=\sigma_{s}^{2}+\sigma_{n}^{2}$. The signal-to-noise ratio is $\left(\sigma_{s} / \sigma_{n}\right)^{2}$, and its decibel value is $10 \log \left(\sigma_{s} / \sigma_{n}\right)^{2}$. [These quantities are equivalent to the commonly used expressions $\Delta I / I$ and $10 \log (\Delta I / I)$, respectively, but in this work we prefer Egan's terminology, partly because it emphasizes that we are measuring Gaussian noise and partly because $\Delta I$ can refer either to the signal power or to the just noticeable difference.] Another ratio that we used is the ratio of the signal plus noise to the noise power, $\left(\sigma_{s n} / \sigma_{n}\right)^{2}$. Its decibel value is $10 \log \left(\sigma_{s n} / \sigma_{n}\right)^{2}$, or alternatively $(\Delta I+I) / I$ and $\left.10 \log [\Delta I+I) / I\right]$. It is also convenient to define $k=\left(\sigma_{n} / \sigma_{s n}\right)^{2}$. We used a single noise source to produce both the noise waveform and the signal-plus-noise waveform. The measured quantities were, therefore, the mean square voltage or power of the noise, $\sigma_{n}^{2}$, and the mean square voltage or power of the signal plus noise, $\sigma_{s n}^{2}$. The power of the signal on its own, $\sigma_{s}^{2}$, was computed from these measured quantities. For the sinusoids, however, $\sigma_{n}^{2}$ and $\sigma_{s n}^{2}$ were the parameters of two probability functions that governed the probability with which the amplitudes of the sinusoids were selected. One probability function determined the amplitudes of the noise sinusoids; the other function determined the amplitudes of the signal-plus-noise sinusoids. Finally, we designated the degrees of freedom in a chi-square distribution by $\nu$, so $\nu=2 W T$.

\section{Signal Levels}

The discriminability of six different signal levels was studied. The signal-plus-noise to noise ratios, $\left(\sigma_{s n} / \sigma_{n}\right)^{2}$, were $3,6,9,12$, 15 , and $18 \mathrm{~dB}$ [these correspond to signal-to-noise ratios, $\left(\sigma_{s} / \sigma_{n}\right)^{2}$, of $0.0,4.7,8.4,11.7,14.9$, and $17.9 \mathrm{~dB}]$. For the sinusoids, the 


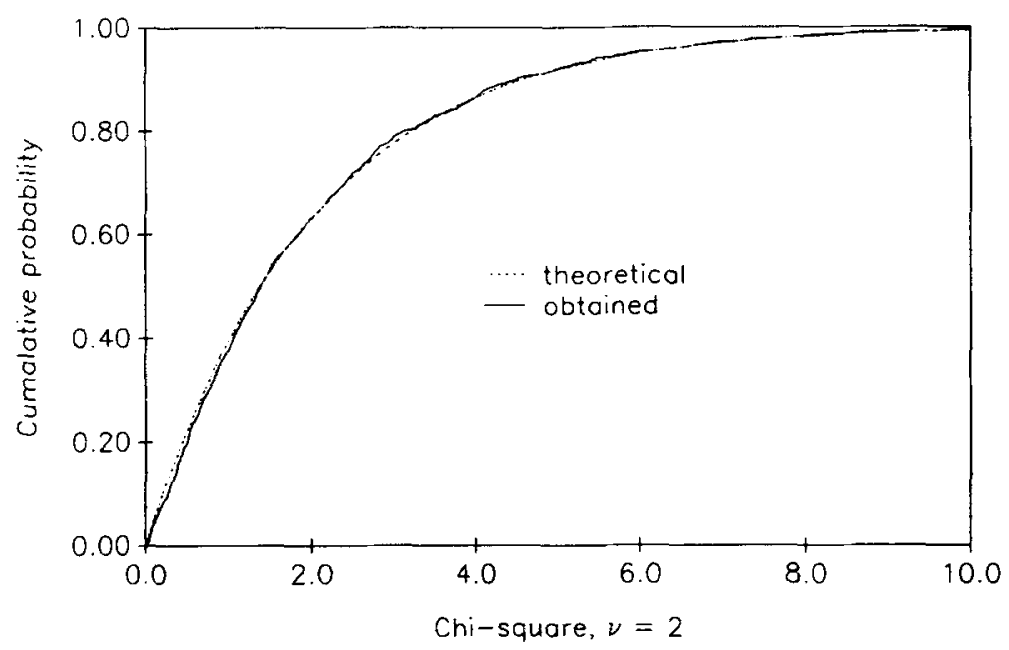

Figure 1. Cumulative probability of 1,021 noise samples with mean square voltages less than the chi-square value shown. The mean of the mean square voltages has been standardized to 2, which is the mean of the chi-square distribution with 2 degrees of freedom; this distribution function is shown by the theoretical curve. The comparison of these two curves was the basis for the Kolmogorov-Smirnov test (see text).

average power of the noise was set to $50 \mathrm{~dB}$ SPL, and for the narrow-band noise it was set to $60 \mathrm{~dB}$ SPL. By this means, the average energy in the two sets of stimuli was the same because the sinusoids were presented for $100 \mathrm{msec}$ (the same value used by Ronken, 1969) and the narrow-band noises for $10 \mathrm{msec}$.

\section{Procedure}

Observers rated their confidence that the signal had been presented during an observation interval by entering 1 of 10 digits (0-9) on a computer keyboard. Every trial began with a 250 -msec warning light. After a 500 -msec pause, the observation interval, marked by a light, occurred, lasting for the duration of the signal. The signal was added to the noise during the observation interval with a probability of .5; otherwise, the noise alone was presented. No trialby-trial feedback was offered, but observers viewed a summary of their results after each block of 200 trials at a given signal level. We adopted this procedure because subjects reported in preliminary tests that trial-by-trial feedback disrupted their performance. (The advantages of providing feedback after a block of trials rather than after every trial are reviewed by Robinson \& Watson, 1972.) Apart from practice, each observer made 800 ratings at each signalto-noise ratio. The six signal-to-noise ratios were presented in a different random order to each observer. The 3 observers who took part in this experiment completed their ratings of the Rayleighdistributed sinusoids first, and then undertook the same task with narrow-band noise. One observer (M.H.) then repeated the experiment with sinusoids, in order to check the stability of the results.

\section{Results}

\section{Psychometric Functions}

Receiver operating characteristics showing hit rate as a function of false-alarm rate were constructed for each subject's ratings at each signal-to-noise ratio. ${ }^{3}$ The area under each ROC, $p(A)$, was computed from the MannWhitney $U$ statistic (Bamber, 1975). This quantity provides a nonparametric index of the discriminability of a signal, and is equal to the proportion correct in a twoalternative forced-choice task (Green \& Swets, 1966).
Figure 2 shows how $p(A)$ depended on the signal-tonoise ratio for each observer. The top panel shows the results for Rayleigh-distributed sinusoids, and the bottom panel for narrow-band noise. The equation for the psychometric function for detecting an increment in a sample of noise is, as Green and McGill (1970) show, the incomplete beta function:

$$
p(A)=\frac{\Gamma(a+b)}{\Gamma(a) \Gamma(b)} \int_{0}^{x} t^{a-1}(1-t)^{b-1} d t, \quad(0 \leq x \leq 1)
$$

where $\Gamma$ is the gamma function and $x=\sigma_{s n}^{2} /\left(\sigma_{s n}^{2}+\sigma_{n}^{2}\right)$. Because the noises differed only in amplitude, their degrees of freedom were equal, so in this case, $a=b$ $=\nu / 2$. When $\nu=2$, the incomplete beta function simplifies to $p(A)=1 /(1+k)$, where $k=\left(\sigma_{n} / \sigma_{s n}\right)^{2}$. The broken line in each panel shows this equation and depicts the psychometric function for an ideal observer for these signals.

The best-fitting degrees of freedom, $\nu$, for the obtained psychometric functions were found from the continued fraction representation of the incomplete beta function (Abramowitz \& Stegun, 1965, p. 944) by means of an algorithm provided in Press, Flannery, Teukolsky, and Vetterling (1986). The solid line in each panel (Figure 2) is a best fit, in a maximum likelihood sense, of a psychometric function based on the incomplete beta function. The fit is to the areas averaged over the 3 observers (excluding the replication by M.H.). Whereas the ideal observer could have achieved the performance (shown by the broken line) for these waveforms with $\nu=2$, these human observers performed as well as an ideal observer that made use of only $1 \mathrm{df}$.

The parameters of the fits for the averaged $p(A)$ values, as well as for each observer, are shown in Table 1 . The fits are not significantly different from the incomplete beta 

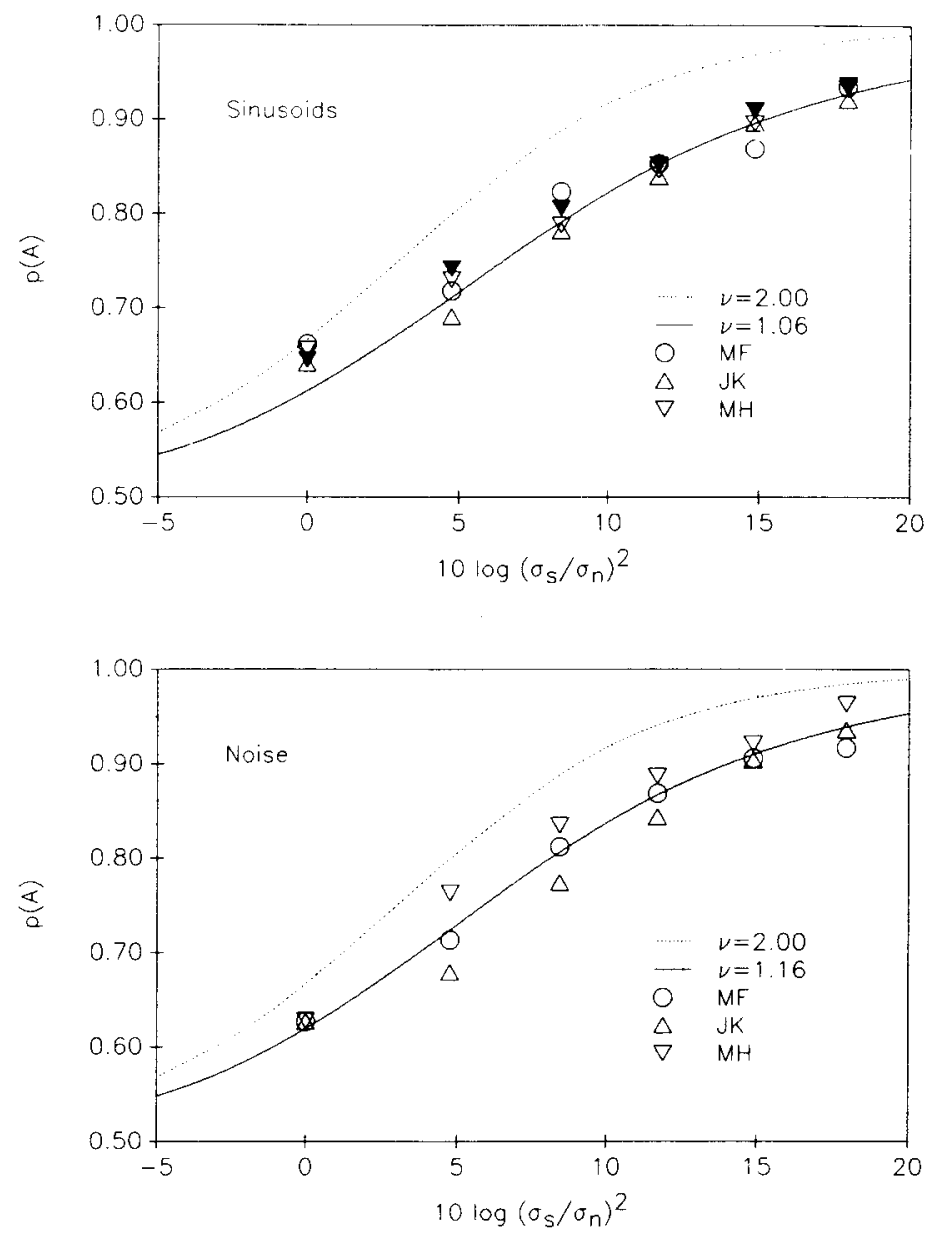

Figure 2. Area under the $\mathrm{ROC}, p(A)$, as a function of signal-to-noise ratio (in decibels) in Experiment 1 . In the top panel, the filled triangles represent a replication of the experiment by observer M.H. The broken lines show the performance of an ideal observer with these signals, and the solid lines show the bestfitting psychometric functions.

function, except for observer M.F., when discriminating amplitude differences in the sinusoids. Table 1 also shows the discrepancy, in decibels, between the position of the ideal observer's psychometric function $(\nu=2)$ and the obtained values of $p(A)$. A unique discrepancy cannot be specified, because the ideal and obtained psychometric functions have different slopes (see Figure 2), and the discrepancy is, therefore, not constant for different signal levels. The decibel values in Table 1 show the amount that the ideal psychometric function must be translated to the right in order to achieve a best fit to the obtained values. The average shift is about $4 \mathrm{~dB}$ for both the sinusoidal and noise signals. Another specification of the shift can be provided by the difference between the ideal and the fitted functions in Figure 2 at the point $p(A)=$ 0.75 ; this difference is $3.4 \mathrm{~dB}$ for the sinusoids and $2.9 \mathrm{~dB}$ for the narrow-band noise.

\section{Receiver Operating Characteristics}

Egan (1975) has shown that ROCs based on narrowband noise with $2 W T=2$ obey a power law in which the hit rate equals the false-alarm rate raised to a power, $k$, where $k=\left(\sigma_{n} / \sigma_{s n}\right)^{2}$, as before. To compare this ideal performance with the obtained results, the data were fitted to nonstandardized chi-square density functions by maximum likelihood procedures and the best-fitting values of $\nu$ and $k$ found for each ROC. (Greig, 1990, has developed a good approximation for this case, but we have used the exact function.)

There were 42 ROCs available for analysis from this experiment ( 3 observers $\times 6$ signal-to-noise ratios $\times 2$ types of signals, sinusoids and narrow-band noise, plus 6 ROCs from M.H.'s replication of the task with sinusoids). Of the 42 fits, 2 ROCs for the narrow-band noise required $\nu>100$ and were, therefore, essentially fitted 
Table 1

Best-Fitting Degrees of Freedom, $v$, for Each Psychometric Function and its Goodness-of-fit Statistic, $21 \mathrm{n} \lambda$, and the Shift in Decibels Required for an Ideal Psychometric Function to fit the Obtained Results

\begin{tabular}{lccccccc} 
& \multicolumn{3}{c}{ Sinusoids } & & \multicolumn{2}{c}{ Noise } \\
\cline { 2 - 4 } \cline { 6 - 7 } Observer & $\nu$ & $2 \ln \lambda$ & Shift & $\nu$ & $21 \mathrm{n} \lambda$ & Shift \\
\hline M.F. & 1.09 & $19.83^{*}$ & 3.9 & 1.11 & 4.97 & 3.9 \\
J.K. & 1.01 & 5.05 & 4.9 & 1.05 & 7.87 & 4.9 \\
M.H. & 1.08 & 8.75 & 4.2 & 1.38 & 3.60 & 2.5 \\
M.H. (rep $\dagger$ & 1.09 & 8.44 & 3.7 & & & \\
Mean $p(A)$ & 1.06 & 7.35 & 4.4 & 1.16 & 0.35 & 3.8 \\
\hline${ }^{*} p<.01$. & †Replication. & & & &
\end{tabular}

by the normal-normal equal-variance model. Of the remaining 40 ROCs, the mean and standard error of $\nu$ for the 24 ROCs for the discrimination of sinusoids was $5.04 \pm 1.05$, and for the 16 ROCs for the discrimination of narrow-band noises it was $7.27 \pm 1.16$. The degrees of freedom for the best-fitting ROCs for both the sinusoids and the narrow-band noise were, on average, significantly larger than the theoretical ideal value of $2(p<.01)$. The value of $k$ decreased with signal-to-noise ratio, as expected.

Figure 3 illustrates 12 of these ROCs, from subject J.K., at each signal-to-noise ratio for both sinusoids (circles) and narrow-band noise (triangles). The top line in each panel shows the ideal ROC for each value of $\left(\sigma_{s n} / \sigma_{n}\right)^{2}$; the other lines are the best-fitting chi-square ROCs for the sinusoids and the narrow-band noise. For the noise at $9 \mathrm{~dB}$, a fit for this model could not be found for this subject (these data illustrate one of the two ROCs that conform to the normal-normal equal-variance model).

\section{EXPERIMENT 2}

We undertook a second experiment in order to check whether the principal results found in Experiment 1 were independent of the average level of the noise stimulus. In Experiment 1, the average level of the noise was always $50 \mathrm{~dB}$ SPL for the sinusoids and $60 \mathrm{~dB}$ SPL for the narrow-band noise. Ronken (1969) found that amplitude resolution of Rayleigh-distributed sinusoids was independent of level over a wide range-a result that is congruent with Weber's law but not with the usual finding for amplitude resolution of sinusoids, in which resolution improves with level.

\section{Method}

Observers

The two authors served as observers.

\section{Apparatus and Signal Levels}

The apparatus and techniques were the same as those used in Experiment 1 . However, only one signal-plus-noise to noise ratio $\left(\sigma_{s n} / \sigma_{n}\right)^{2}$, was presented for discrimination: $9 \mathrm{~dB}$. The signal-tonoise ratio $\left(\sigma_{s} / \sigma_{n}\right)^{2}$ was therefore $8.4 \mathrm{~dB}$. The main variable of the experiment was the average level of the noise. For the sinusoids, the levels studied were $30,40,50$, and $60 \mathrm{~dB}$ SPL; for the narrowband noise they were $40,50,60$, and $70 \mathrm{~dB}$ SPL. Again, the sinusoids were presented for $100 \mathrm{msec}$ and the noises were presented for $10 \mathrm{msec}$ so that the energies in the two sets of stimuli were identical

\section{Procedure}

The rating procedure was the same as that used in Experiment 1. Apart from practice, each observer made 600 ratings at each noise level for each stimulus set-sinusoids and narrow-band noises. The levels were presented in a different random order to each observer, and the first 50 trials of a block of 200 trials were regarded as warmup trials and were discarded from analysis. Each observer undertook two blocks at one level before moving to the next assigned level, and then repeated the levels in reverse order to complete 600 trials per level, excluding warm-up trials.

\section{Results}

\section{Effect of Noise Level}

Figure 4 shows how the average level of the noise affected the area under the ROC, $p(A)$, for each stimulus type for each observer. Evidently, there is no systematic effect of level on $p(A)$; in other words, Weber's law holds. The near miss to Weber's law on these coordinates is shown by a systematic increase in $p(A)$ with noise level. The line illustrating the near miss is derived from Equation 3.9 of Green's (1988) summary of the evidence on the near miss (see also Laming, 1986, Equation 1.10, p. 14). The relation is: $p(A)=\Phi\left[\left(4 \Delta A \cdot A^{-5 / 6}\right) / \sqrt{2}\right]$, where $\Delta A$ is the just noticeable difference in amplitude and $\Phi$ is the cumulative normal distribution.

\section{Receiver Operating Characteristics}

Figure 5 shows the ROCs obtained in this experiment for each observer; the top panels show the results for the sinusoids, and the bottom panels show the results for the narrow-band noises. The ideal characteristic shown by the curve is a power function, $h=f^{k}$, where $h$ is the hit rate, $f$ is the false-alarm rate, and $k=\left(\sigma_{n} / \sigma_{s n}\right)^{2}=0.126$. This ideal operating characteristic is derived from nonstandardized chi-square density functions with $2 \mathrm{df}$.

The data for each ROC were fitted by maximum likelihood methods to chi-square densities to find the best-fitting values of $\nu$ and $k$. The results are shown in Table 2. None of the fits is significantly different from the model at the .01 level, although two fail the fit at the less stringent criterion of .05 . For the sinusoids, the average of the bestfitting degrees of freedom for J.I. was 2.31 , whereas for M.H. it was 3.22. For the narrow-band noises, the fits showed large variations from one noise level to another, particularly for M.H. In general, M.H.'s ROCs were less asymmetric about the negative diagonal for the narrowband noise than for the sinusoids.

\section{DISCUSSION}

\section{Comparison of Sinusoids and Narrow-band Noise}

First, we noted that there was little difference between the results for the Rayleigh-distributed sinusoids and the narrow-band noise in our experiments. However, as gratifying as this may be, it leaves unanswered the origin of the difference between Ronken's (1969) data and those of Whitmore et al. (1968). In the present study, both the shapes and the locations of the psychometric functions fitted to each type of stimulus were quite similar (Experi- 

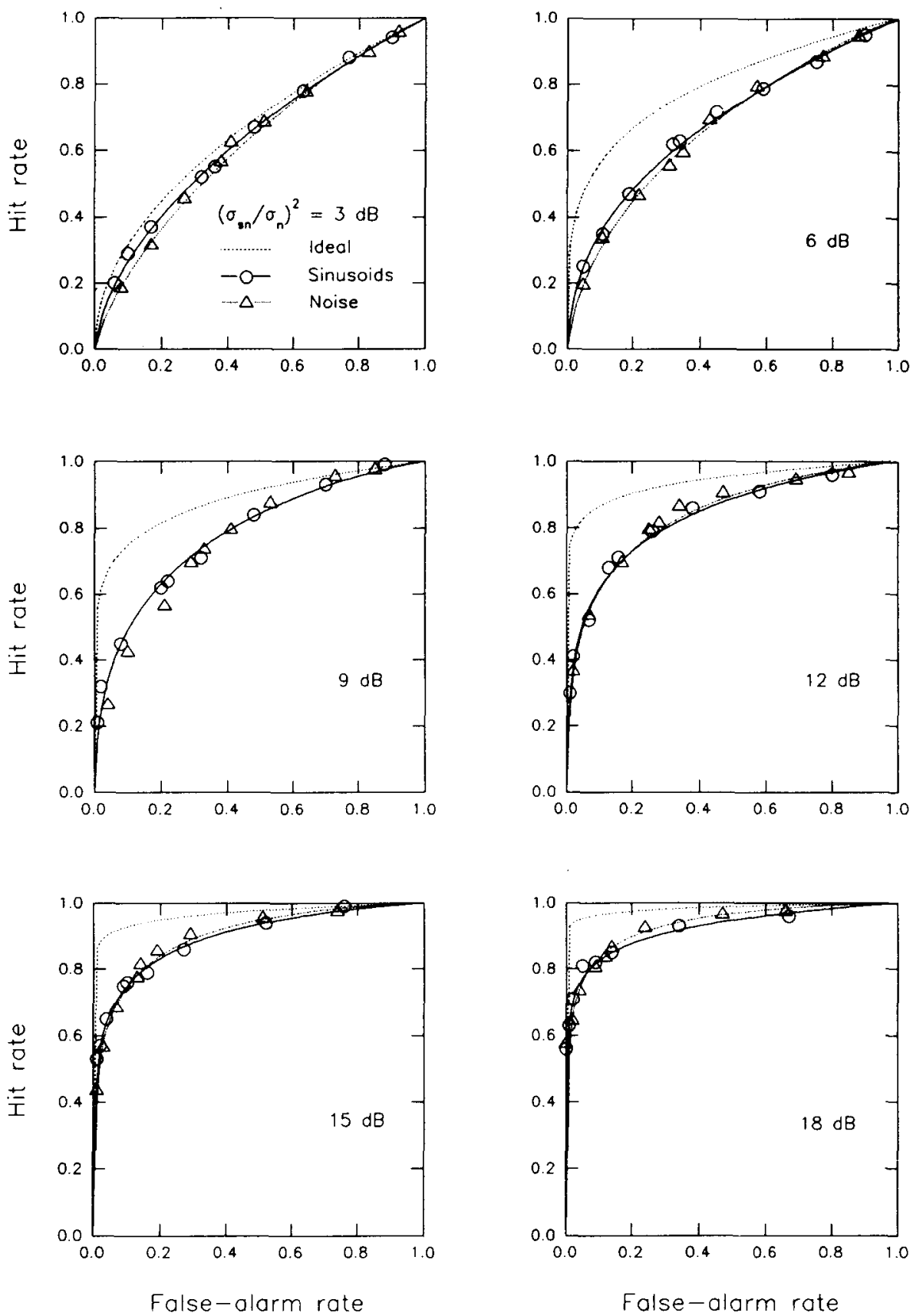

Figure 3. ROCs for subject J.K. in Experiment 1. Each panel is for a different value of $10 \log \left(\sigma_{s n} / \sigma_{n}\right)^{2}$; the circles represent the results for the sinusoids, and the triangles the results for the noises. The top line shows the performance of an ideal observer for each signal, and the solid and dotted lines indicate the best-fitting chi-square ROCs for the sinusoids and narrow-band noises, respectively.

ment 1 , Figure 2 and Table 1). There were also no obvious differences as a function of sound pressure between the performance of the observers on these two signal types (Experiment 2, Figure 4). The shape of the ROCs in Figure 5 may reveal a difference between the results for sinusoids and the results for narrow-band noise. Observer M.H. produced more symmetrical ROCs when discriminating amplitudes of narrow-band noise than when dis- criminating amplitudes of Rayleigh-distributed sinusoids, although his performance in this respect was quite variable (see Table 2).

\section{Psychometric Functions}

There was a discrepancy on the average of about $4 \mathrm{~dB}$ between the location of the psychometric function of an ideal observer and that of the observers in Experiment 1 


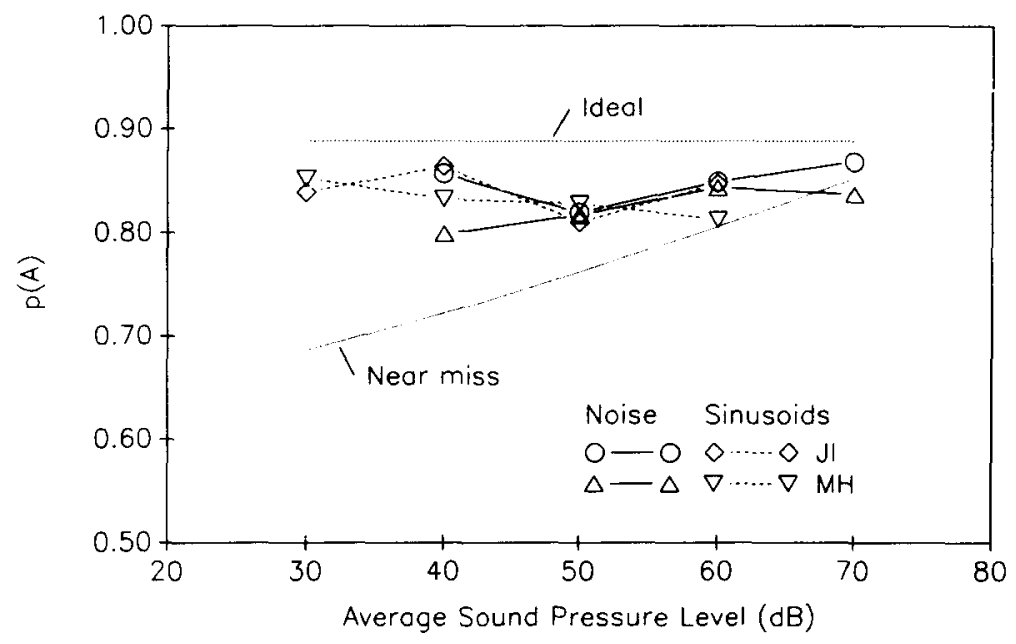

\begin{abstract}
Figure 4. Area under the $\mathrm{ROC}, p(A)$, as a function of average sound pressure level for each class of signal in Experiment 2. The signal-to-noise ratio was the same at each sound pressure level, and the performance of an ideal observer is shown by the horizontal line. The line for the near miss to Weber's law has been scaled so that the point at $70 \mathrm{~dB}$ SPL coincides with the mean of the obtained results.
\end{abstract}

(Table 1). This is much more than the $0.7 \mathrm{~dB}$ reported by Ronken (1969), but within the range found by Whitmore et al. (1968). Some of the difference between our result and Ronken's is attributable to the difference in the dimension he used, $10 \log \left(\sigma_{s n} / \sigma_{n}\right)^{2}$, and the dimension in Figure 2, $10 \log \left(\sigma_{s} / \sigma_{n}\right)^{2}$. These two dimensions depend upon the parameter $k$, and $10 \log \left(\sigma_{s n} / \sigma_{n}\right)^{2}$ varies with $10 \log \left(\sigma_{s} / \sigma_{n}\right)^{2}$ as:

$$
\frac{\mathrm{dy}}{\mathrm{dk}} \mid \frac{\mathrm{dx}}{\mathrm{dk}}=1-k . \quad(0 \leq k \leq 1)
$$

Thus, Ronken's $0.7 \mathrm{~dB}$ amounts to about $1.0 \mathrm{~dB}$ on the coordinates of Figure 2 . Some of the remaining difference can be explained by noting that the discrepancy at $p(A)=0.75$ (the value reported by Ronken) is somewhat less than the average discrepancy of $4 \mathrm{~dB}$. For our data, this value is $3.2 \mathrm{~dB}$ on the average. This leaves a difference of about $2.2 \mathrm{~dB}$ between our result and Ronken's. However, we conjecture that, despite the theoretical equivalence between proportion correct and $p(A)$, the single-interval design we adopted may underestimate proportion correct in a two-interval forced-choice experiment. In support of this conjecture, we note that Viemeister (1970) compared rating and forced-choice methods for measuring intensity discrimination of sinusoids and found that the index, $d^{\prime}$, for forced choice was greater than that for rating by a factor of approximately 2 , rather than the theoretical factor of $\sqrt{2}$. Jesteadt and Bilger (1974) also found that a two-interval forced-choice procedure yielded higher indices of performance than did those from a theoretically equivalent single-interval procedure. Thus, Ronken's result and ours may not be so disparate after all.

In Figure 2, the data are reasonably well described by the incomplete beta psychometric function with $\nu=1$ (see also Table 1). Green and McGill (1970) pointed out that performance in this case is theoretically based on a single instantaneous sample of the waveform during the observation interval. Whereas there are two independent samples available in the waveform, the human observers do about as well as an ideal observer using only one of those samples. The shape of our obtained psychometric function, however, differs from that found by Whitmore et al. (1968) because, in their case, the shape of the psychometric function was the same as that of the ideal function, though shifted in position.

\section{Shape of the ROCs}

The shapes of the ROCs we obtained are congruent with our findings for the shape of the psychometric functions. The ROCs were fitted by chi-square probability densities with two or three times (and occasionally much more) the number of degrees of freedom actually present. Whereas the fits in Figure 2 show that the ideal observer requires fewer degrees of freedom than do human observers, the fits in Figures 3 and 5 show that human observers require more degrees of freedom than the ideal observer. In other words, the subjects performed as though additional noise were added to the signals.

\section{Judgmental or Internal Noise}

One way of accounting for the difference between ideal and human observers, therefore, is to suppose that an additional component of noise is entailed in human judgment. Whereas the ideal observer is faced only with the variance associated with the stimulus - the signal noisehumans also have to contend with judgmental noise, or more generally, internal noise. Durlach and Braida (1969) 

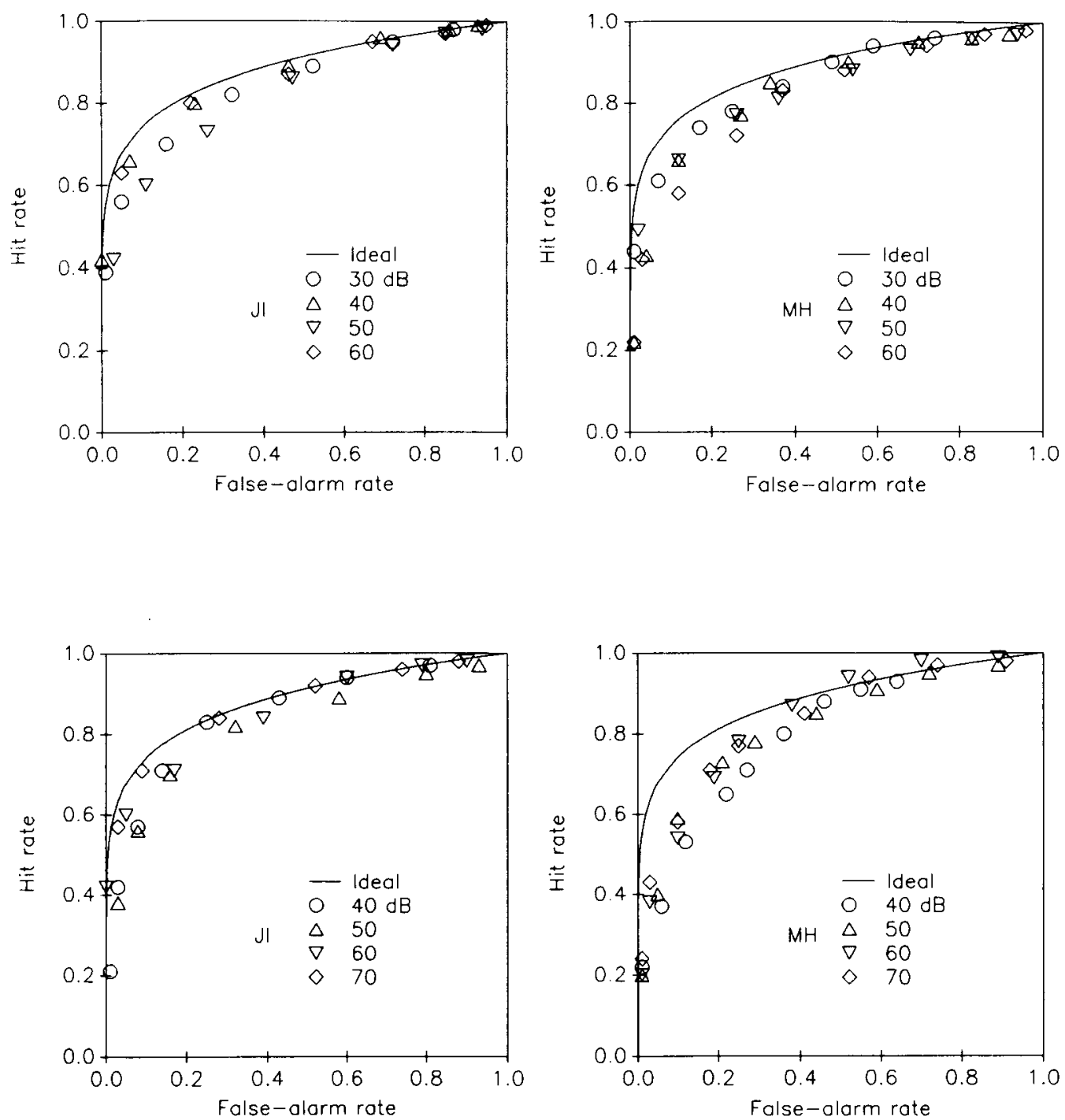

Figure 5. ROCs at each sound pressure level for sinusoids (top panels) and narrow-band noise (bottom panels) for each observer in Experiment 2. The curve in each panel represents the ROC of an ideal observer for this experiment.

and Laming (1984) offer models of how this process might degrade performance relative to that attainable with signal noise alone.

There have been several attempts to assess the size of an observer's internal noise relative to the stimulus noise (e.g., Spiegel \& Green, 1981). A convenient summary of these results would be to say that the ratio of internal to stimulus noise is near unity. Ronken (1969), however, estimated a ratio of 0.3 from his results. This lower than usual estimate (which was based on only one point of the psychometric function) reflects the closeness of his observers' results to ideal performance. The problem with Ronken's estimate is that it varies with the signal-to-noise ratio at which it is made.

To see this for our results, first note that, for the ideal observer, $\left(\sigma_{s n} / \sigma_{n}\right)^{2}=1 / k=p(A) /[1-p(A)]$. For the hu- man observer, Ronken (1969) postulated that internal noise, $\sigma_{i}^{2}$, adds to the stimulus noise, $\sigma_{n}^{2}$, to degrade performance, so in that case $\left(\sigma_{s n}^{2}+\sigma_{i}^{2}\right) /\left(\sigma_{n}^{2}+\sigma_{i}^{2}\right)=$ $p(A) /[1-p(A)]$. Next, observe that our data are reasonably well approximated by the incomplete beta function for $\nu=1$ (see Figure 2). For this case, the function reduces to $p(A)=1-2 \cdot \arctan (\sqrt{k}) / \pi$, and this can therefore be taken as a representation of our data. From this, it can be deduced that $\sigma_{i}^{2}$ is related to $\sigma_{n}^{2}$ by the factor

$$
\frac{\cos [\pi p(A)]+2 p(A)-1}{\{\cos [\pi p(A)]+1\}[1-2 p(A)]}
$$

which is a function of $p(A)$. Therefore, $\sigma_{i}^{2}$ is not a constant ratio of $\sigma_{n}^{2}$, but depends on the point at which it is taken. Our results, nevertheless, can be compared with 
Table 2

Best-Fitting Parameters, $\nu$ and $k$, for a Chi-Square ROC and Their Goodness-of-fit Statistic, 21n $\lambda$, Found in Experiment 2

\begin{tabular}{|c|c|c|c|c|c|c|}
\hline \multirow{3}{*}{$\begin{array}{c}\text { Decibels } \\
\text { (SPL) }\end{array}$} & \multicolumn{6}{|c|}{ Subjects } \\
\hline & \multicolumn{3}{|c|}{ J.I. } & \multicolumn{3}{|c|}{ M.H. } \\
\hline & $\nu$ & $k$ & $2 \ln \lambda$ & $\nu$ & $k$ & $2 \ln \lambda$ \\
\hline \multicolumn{7}{|c|}{ Sinusoids } \\
\hline 30 & 2.61 & .24 & 1.57 & 3.03 & .25 & 3.87 \\
\hline 40 & 2.53 & .20 & 2.20 & 4.31 & .37 & 18.34 \\
\hline 50 & 2.33 & .26 & 6.61 & 1.48 & .14 & 6.71 \\
\hline 60 & 1.78 & .14 & 6.46 & 4.05 & .38 & 11.09 \\
\hline \multicolumn{7}{|c|}{ Noise } \\
\hline 40 & 14.75 & .56 & 6.72 & 56.77 & .79 & 6.63 \\
\hline 50 & 2.89 & .30 & $12.70^{*}$ & 5.76 & .45 & 6.74 \\
\hline 60 & 1.93 & .17 & 7.10 & 45.37 & .74 & 8.80 \\
\hline 70 & 2.52 & .18 & 0.83 & 7.43 & .47 & 6.47 \\
\hline
\end{tabular}

Ronken's estimate of internal noise at $p(A)=0.75$. For this point, the above factor turns out to be $\sqrt{2}$, a factor that is considerably larger than Ronken's estimate of 0.3 .

The discrepancies between the obtained and ideal ROCs, shown in both Figures 3 and 5, are qualitatively consistent with the above analysis of the discrepancies between the psychometric functions shown in Figure 2. The fits of the ROCs again show that, given signals with $2 \mathrm{df}$, the human observers do not perform at the level of the ideal observer. In this case, the model shows not how many fewer degrees of freedom an ideal observer would need to match the performance of the human observers, but how many more degrees of freedom would be required to produce the more symmetrical ROCs that the human observers produce. The added degrees of freedom can be conceptualized as added internal noise that is not present in the signals. However, a quantitative estimate of the magnitude of the added internal noise required to degrade performance to match the obtained performance is more difficult to determine in this case. This is due to the effect of the second fitted parameter, $k$, which interacts with the effect of the fitted degrees of freedom, $\nu$. It may be possible to estimate the level of internal noise required to produce the obtained ROCs by using the characteristic functions of the distributions involved, but so far we have not been able to solve this problem.

An alternative strategy has been advanced by Taylor, Boven, and Whitmore (1991). Instead of attempting to estimate the magnitude of internal noise, they remove the additional noise by averaging it out. This can be accomplished provided identical samples of signal noise are presented several times. The averaging then eliminates the additional noise because it is assumed to be unique to each judgment. The signal noise, being constant from trial to trial, alone remains. We cannot perform this analysis on our data because we did not present identical signals on several trials. In any event, our obtained ROCs show the performance of human observers, imperfections and all.
Since our purpose was to compare human performance with a theoretical ideal, the additional noise evidently entailed in the discrimination of Rayleigh noises must form part of that comparison.

\section{Weber's Law and Rayleigh Noise}

There is no convincing evidence for the near miss to Weber's law in the results shown in Figure 4. The near miss would be manifested by an improvement in performance with sound pressure on these coordinates, as shown by the line labeled "near miss." In this respect, our results are in accord with Ronken's (1969) findings. The limits of the ear's capacity to resolve the difference in amplitude of sinusoids of fixed amplitude are not reached in studies that employ Rayleigh noise, and it is at these limits where the near miss to Weber's law can be expected.

\section{Summary}

The findings of this study are as follows:

1. Discrimination of the amplitude of Rayleighdistributed sinusoids and of narrow-band Rayleigh noise yielded similar results. However, the ROCs for the narrow-band noise may have been more symmetrical (based on chi-square densities with more degrees of freedom) than the ROCs for sinusoids.

2. The psychometric functions obtained for Rayleighdistributed sinusoids and for narrow-band Rayleigh noise differed from those of an ideal observer, both in shape and location. The shapes of the obtained functions were the same as those of an ideal observer using only $1 \mathrm{df}$ rather than the available $2 \mathrm{df}$. The location of the obtained functions was displaced, on average, about $4 \mathrm{~dB}$ from the ideal function when measured in units of $10 \log \left(\sigma_{s} / \sigma_{n}\right)^{2}$, and about $3 \mathrm{~dB}$ from the ideal function at the point $p(A)=0.75$.

3. Most of the ROCs were not significantly different from those of a chi-square observer. However, the degrees of freedom required to fit the ROCs were usually two or three times the theoretical value, and occasionally much more.

4. Weber's law, not the near miss, held for the discrimination of the amplitude of these sinusoids and narrowband noises.

\section{REFERENCES}

Abramowitz, M., \& Stegun, I. A. (1965). Handbook of mathematical functions. New York: Dover.

BAMBER, D. (1975). The area above the ordinal dominance graph and the area below the receiver operating characteristic graph. Journal of Mathematical Psychology, 12, 387-415.

Durlach, N. I., Braida, L. D. (1969). Intensity perception. I. Preliminary theory of intensity resolution. Jourmal of the Acoustical Society of America, 46, 372-383.

EGAN, J. P. (1975). Signal detection theory and ROC analysis. New York: Academic Press.

GREEN, D. M. (1960). Auditory detection of a noise signal. Journal of the Acoustical Society of America, 32, 121-131. 
GreEn, D. M. (1988). Profile analysis: Auditory intensity discrimination. New York: Oxford University Press.

GreEN, D. M., \& McGiLL, W. J. (1970). On the equivalence of detection probabilities and well-known statistical quantities. Psychological Review, 77, 294-301.

Green, D. M., SWets, J. A. (1966). Signal detection theory and psychophysics. New York: Wiley.

GREIG, G. L. (1990). On the shape of energy-detection ROC curves. Perception \& Psychophysics, 48, 77-81.

IRWIN, R. J. (1989). Psychometric functions for the discrimination of differences in intensity of Gaussian noise. Quarterly Journal of Experimental Psychology, 41A, 655-674.

JESTEADT, W., BLLGER, R. C. (1974). Intensity and frequency discrimination in one- and two-interval paradigms. Joumal of the Acoustical Society of America, 55, 1266-1276.

JesteAdT, W., Wier, C. C., GREeN, D. M. (1977). Intensity discrimination as a function of frequency and sensation level. Journal of the Acoustical Society of America, 61, 169-177.

Laming, D. (1984). The relativity of "absolute" judgements. British Journal of Mathematical and Statistical Psychology, 37, 152-183.

Laming, D. (1986). Sensory analysis. London: Academic Press.

Press, W. H., Flannery, B. P., Teukolsky, S. A., \& Vetterling, W. T. (1986). Numerical recipes: The art of scientific computing. Cambridge: Cambridge University Press

Robinson, D. E., \& Watson, C. S. (1972). Psychophysical methods in modern psychoacoustics. In J. V. Tobias (Ed.), Foundations of modern auditory theory (Vol. 2, pp. 101-131). New York: Academic Press.
RONKEN, D. A. (1969). Intensity discrimination of Rayleigh noise. Journal of the Acoustical Society of America, 45, 54-57.

SPIEGEL, M. F., Green, D. M. (1981). Two procedures for estimating internal noise. Joumal of the Acoustical Society of America, 70 , 69-73.

TAYlor, A., Boven, R., \& Whitmore, J. (1991). Reduction of unique noise in the psychophysics of hearing by Group Operating Characteristic analysis. Psychological Bulletin, 109, 133-146.

VIEMEISTER, N. F. (1970). Intensity discrimination: Performance in three paradigms. Perception \& Psychophysics, 8, 417-419.

Whitmore, J., Williams, P. I., \& ERmey, H. L. (1968, May). Psychometric function from Rayleigh-Rayleigh ROC curves. Paper presented at the 75th meeting of the Acoustical Society of America, Ottawa, Ontario, Canada.

WOODWORTH, R. S., SCHLOSBERG, H. (1954). Experimental psychology. New York: Hoit.

\section{NOTES}

1. We are grateful to Donald Laming for pointing this out (letter of August 21, 1989).

2. More precisely, the upper bound of the last bin was placed to encompass 0.9995 of the area under the density function, a value where $A=\sigma \sqrt{\left[\ln \left(4 \times 10^{6}\right)\right]}$, and the 20 amplitudes were equally spaced between zero and that value.

3. Occasionally, no ratings were made in the extreme categories for some signal-to-noise ratios. The empty categories were then combined with adjacent ones

(Manuscript received July 24, 1991;

revision accepted for publication January 16, 1992.) 